\title{
Introdução aos números transcendentes e aos números de Liouville
}

\author{
Lindauriane Vieira
}

Luiz Feitoza

Diego Lima

Genilson da Silva

\section{Resumo}

A Teoria dos Números Transcendentes é uma área que foi originada por Joseph Liouville em seu ilustre trabalho de 1844 (ver [4]), no qual ele criou uma classe numérica, os Números de Liouville, pela qual não só provou a existência dos números transcendentes, como também explicitou seus primeiros exemplos. Nesse sentido, este trabalho tem como objetivo desenvolver um estudo sobre a Teoria dos Números Transcendentes, especificamente dos Números de Liouville. Esse estudo foi realizado através de uma pesquisa de teor qualitativo, constituindo-se em um estudo bibliográfico. Dentre os principais resultados, destacam-se a definição e a caracterização do conjunto dos números algébricos e dos números transcendentes, e a apresentação de importantes resultados sobre a classe dos Números de Liouville. Nessa perspectiva, este trabalho apresenta-se como uma pequena "pesquisa" em Teoria Transcendente, cuja importância reside em subsidiar estudos futuros de modo que coadjuve o seu progresso teórico no meio científico, haja vista que é uma área recente e que tem despertado o interesse de muitos pesquisadores.

Palavras-chave: Números Algébricos; Números Transcendentes; Números de Liouville.

\begin{abstract}
The Theory of Transcendental Numbers is an area that was originated by Joseph Liouville in his illustrious work of 1844, in which he created a numerical class, the Liouville Numbers, by which he not only proved the existence of transcendental numbers, as well as his first examples. In this sense, this work aims to develop a study on the Theory of Transcendental Numbers, specifically the Liouville Numbers. This study was carried out through a qualitative research, constituting a bibliographic study. Among the main results, we highlight the definition and characterization of the set of algebraic numbers and transcendental numbers, and the presentation of important results on the class of Liouville Numbers. In this perspective, this work presents itself as a small "research" in Transcendental Theory, whose importance lies in subsidizing future studies in a way that supports its theoretical progress in the scientific environment, since it is a recent area that has aroused interest of many researchers.
\end{abstract}

Keywords: Algebraic Numbers; Transcendental Numbers; Liouville Numbers. 


\section{Introdução}

A Teoria dos Números é um ramo da Matemática que se dedica ao estudo de teorias que circundam os números imersos aos conjuntos numéricos (Naturais, Inteiros, Racionais, Irracionais, Reais e Complexos). Da vasta área abrangida pela teoria dos números, evidenciamos particularmente a Teoria dos Números Transcendentes.

A referida área foi apresentada por Joseph Liouville em seu trabalho de 1844, no qual ele obteve, pela primeira vez, uma classe de números que não satisfazem nenhuma equação algébrica com coeficientes inteiros. A concepção de Liouville para construir os números transcendentes foi desenvolver uma propriedade que seja satisfeita por todos os números reais algébricos irracionais: se $\alpha \in \mathbb{R}$ é algébrico irracional de grau $n$, então existe uma constante $A>0$ (constante não universal, pois depende de $\alpha$ ), tal que $\left|\alpha-\frac{p}{q}\right|>\frac{A}{q^{n}}$ para todo $\frac{p}{q} \in \mathbb{Q}$. Dessa forma, qualquer número real irracional que não satisfaça tal propriedade é transcendente.

Utilizando o fato acima, em 1851 (ver [5]), Liouville provou a transcendência dos, ulteriormente assim chamados, números de Liouville: um número real $\alpha$ é chamado número de Liouville se existe uma sequência de racionais distintos $\left\{\frac{p_{j}}{q_{j}}\right\}_{j \geq 1}$, tal que $\left|\alpha-\frac{p_{j}}{q_{j}}\right|<\frac{1}{q_{j}^{j}}$. A partir dessa propriedade, Liouville exibiu o primeiro número transcendente da história: $l=\sum_{k=1}^{\infty} 10^{-k !}$, conhecido como a Constante de Liouville.

Matemáticos famosos contribuíram para o desenvolvimento do estudo da classe dos números transcendentes. Dentre eles, Charles Hermite que, em 1873, demonstrou a transcendência de $e$. Ferdinand von Lindemann, em 1882, ampliou a demonstração de que é transcendente e mostrou que $\pi$ é transcendente.

Posteriormente, Alexander Gelfond, em 1934, e Theodor Schneider, em 1935, resolveram independentemente o famoso $7^{\mathrm{O}}$ Problema de Hilbert sobre a transcendência de números da forma $\alpha^{\beta}$, sendo $\alpha$ um número algébrico (diferente de zero e um), e $\beta$ um número algébrico (não racional), especificamente para o caso particular do número $2^{\sqrt{2}}$. O curioso é que para potências de números transcendentes por transcendentes, ainda não existe nenhum resultado parecido com o de Gelfond e Schneider, mas sabemos que tais potências tanto podem resultar em um número algébrico como em um número transcendente.

Existe no estudo da Teoria Transcendente um forte paradoxo que pode ser encontrado em [8]: se quase todos os números são transcendentes, por que demonstrar a transcendência de um número é, via de regra, algo tão complexo e desafiador? A principal obstrução é que um número transcendente é definido não pelo que ele é, mas, em vez disso, pelo que ele não é. Por esse motivo, perdura-se na atualidade uma série de números em que não se conhece nada acerca de sua natureza como, $e+\pi$ e $e \pi$.

À vista disso, este trabalho tem como objetivo desenvolver um estudo sobre a Teoria dos Números Transcendentes, especificamente dos números de Liouville. Esse estudo foi desenvolvido por meio de uma pesquisa qualitativa, caracterizando-se por um estudo bibliográfico.

\section{Números algébricos e transcendentes}

Iniciaremos esta seção apresentando o conjunto dos números algébricos e, por meio desse, chegaremos a um novo conjunto numérico, o conjunto dos números chamados de transcendentes. Além 
disso, apresentaremos a caracterização de ambos os conjuntos.

\subsection{Definições e Exemplos}

Definição 1. Qualquer solução real ou complexa de toda equação polinomial da forma:

$$
a_{n} x^{n}+a_{n-1} x^{n-1}+\cdots+a_{1} x+a_{0}=0
$$

onde $n \in \mathbb{N}, a_{i} \in \mathbb{Q}$ para todo $i=1,2,3, \ldots, n, a_{n} \neq 0$ é chamado de número algébrico. Os algébricos são o feixo algébrico de $\mathbb{Q}$, isto é, são o conjunto das raízes de todos os polinômios com coeficientes racionais, por isso denotamos os mesmos por $\overline{\mathbb{Q}}$.

Exemplo 1. Todo número racional é algébrico, pois temos que o número da forma $\frac{p}{q} \operatorname{com} p, q \in \mathbb{Z}$, $q \neq 0$ é raiz do polinômio $q x-p$. Entretanto, nem todo número algébrico é racional, já que $\sqrt{2}$ é algébrico (pois é raiz do polinômio $x^{2}-2$ ) e não é racional.

Observação 1. Posto o exemplo acima, evidenciamos o fato de que o conjunto dos números racionais está contido no conjunto dos números algébricos.

Exemplo 2. $\sqrt{2}+\sqrt[3]{2}$ é um número algébrico pois é solução da equação

$$
x^{6}-6 x^{4}-6 x^{3}+12 x^{2}-36 x+1=0 .
$$

Definição 2. Os números reais (ou complexos) que não são algébricos denominam-se de transcendentes.

A rigor de notação utilizamos $\mathbb{T}$ reais.

A definição de número transcendente é do século XVIII e, segundo Leonard Euler, esses números são chamados transcendentes porque "transcendem" o poder das operações algébricas [3]. Todavia, em 1844 Joseph Liouville (1809-1882) exibiu o número: $\sum_{k=1}^{\infty} 10^{-k !}$, o primeiro a ter sua transcendência demonstrada.

Observação 2. As definições acima nos dão uma classificação dos reais como a união disjunta dos números algébricos reais e transcendentes reais.

\subsection{Caracterização dos Algébricos e Transcendentes}

Iniciaremos com o teorema que enuncia algumas propriedades do conjunto dos números algébricos.

Teorema 1. Dados $\alpha, \beta \in \overline{\mathbb{Q}}$, temos:

(i) $\alpha \pm \beta \in \overline{\mathbb{Q}}$;

(ii) $\alpha \cdot \beta \in \overline{\mathbb{Q}}$;

(iii) $\alpha \neq 0$, ent $\tilde{a} o \alpha^{-1} \in \overline{\mathbb{Q}}$.

Demonstração. Ver [2]. 
Usando o teorema anterior verifica-se que o conjunto dos números algébricos forma um corpo.

Ao longo deste trabalho, veremos alguns exemplos de números transcendentes. Entretanto, é possível verificar a existência desses números sem apresentar exemplos explícitos, utilizando o seguinte teorema.

Teorema 2. O conjunto $\overline{\mathbb{Q}}$ dos números algébricos é enumerável.

Demonstração. Dado um polinômio com coeficientes inteiros

$$
P(x)=a_{n} x^{n}+a_{n-1} x^{n-1}+\cdots+a_{1} x+a_{0},
$$

onde $n \in \mathbb{N}$ e $a_{n} \neq 0$.

Definimos sua altura como sendo o número natural

$$
|P|=\left|a_{n}\right|+\left|a_{n-1}\right|+\cdots+\left|a_{1}\right|+\left|a_{0}\right|+n .
$$

Isto é, a soma dos valores absolutos dos seus coeficientes inteiros, mais o grau do polinômio.

O Teorema Fundamental da Álgebra diz-nos que $P(x)=0$, com $P(x)$ dado em (1), tem exatamente $n$ raízes complexas (contando suas multiplicidades). Sendo que todas, algumas ou nenhuma delas podem ser reais. Isso posto, para um $n$ fixado, o número de polinômios do tipo (1) com uma dada altura é apenas um número finito. Para essa afirmação veja que se tem a inclusão da parcela $n$ na definição da altura descrita em (2). Destarte, as raízes de todos os polinômios de todas as alturas formam um conjunto enumerável de conjuntos finitos. Logo, podemos inferir que o conjunto de todos os números algébricos é enumerável.

Esse teorema foi demonstrado pela primeira vez em 1874, por Cantor, o que foi surpreendente: a enumerabilidade do conjunto dos números algébricos implicaria a existência de uma quantidade infinitamente maior de transcendentes do que algébricos.

Teorema 3. Existem números transcendentes e, mais ainda, o conjunto dos números transcendentes não é enumerável.

Demonstração. Sabemos que o conjunto $\mathbb{R}$, como apresentado na Observação 2, pode ser considerado como a união disjunta do conjunto dos números algébricos reais com o conjunto dos números transcendentes reais. Dessarte, por meio do Teorema 2 segue-se que o conjunto dos algébricos reais é enumerável. Sabendo, também, que o conjunto $\mathbb{R}$ não é enumerável, então o conjunto dos transcendentes reais não é enumerável, já que, caso contrário, $\mathbb{R}$ seria enumerável como união de dois conjuntos enumeráveis. Dessa forma, existe um conjunto infinito não enumerável de números transcendentes.

Observação 3. Esse teorema subsidia o fato de que a cardinalidade do conjunto $\mathbb{T}$ é maior do que a cardinalidade do conjunto $\overline{\mathbb{Q}}$ dos números algébricos. Em outras palavras, isso significa que "existem muito mais números transcendentes do que números algébricos".

Proposição 1. O conjunto $\overline{\mathbb{Q}}$ é denso em $\mathbb{R}$. 
Demonstração. Basta observar, como já apresentado na Observação 1, o fato de que $\overline{\mathbb{Q}}$ contém $\mathbb{Q}$, e que $\mathbb{Q}$ é denso em $\mathbb{R}$, então segue trivialmente que $\overline{\mathbb{Q}}$ é denso em $\mathbb{R}$.

Proposição 2. O conjunto Té denso em $\mathbb{R}$.

Demonstração. Seja $I$ um intervalo aberto não degenerado em $\mathbb{R}$. Se $I$ não contém números transcendentes, então esse seria formado apenas por números algébricos, isto é, seria enumerável. Isso não pode ocorrer, pois todo intervalo não degenerado de números reais não é enumerável. Por conseguinte, o conjunto dos números transcendentes é denso em $\mathbb{R}$.

Agora, vejamos alguns resultados de operações elementares agindo em números algébricos e transcendentes.

Proposição 3. Sejam $\alpha \in \overline{\mathbb{Q}} e \beta \in \mathbb{T}$, então:

(i) $\alpha+\beta \in \mathbb{T}$;

(ii) $\alpha \beta \in \mathbb{T}(\alpha \neq 0)$;

(iii) $\beta^{-1} \in \mathbb{T}$.

Demonstração. (i)Suponha, por contradição, que $\alpha+\beta \in \overline{\mathbb{Q}}$. Então, pelo Teorema 1, $\beta$ será algébrico, o que é uma contradição. Dessa forma, $\alpha+\beta \in \mathbb{T}$.

(ii) Suponha que $\alpha \beta=c \in \overline{\mathbb{Q}}$, como $\alpha \neq 0$, então pelo Teorema $1, \beta=c \cdot \frac{1}{\alpha} \in \overline{\mathbb{Q}}$, o que é um absurdo. Logo, $\alpha \beta \in \mathbb{T}$.

(iii) Supondo $\beta^{-1} \in \overline{\mathbb{Q}}$, então pelo Teorema $1, \frac{1}{\beta^{-1}} \in \overline{\mathbb{Q}}$, mas por outro lado, $\frac{1}{\beta^{-1}}=\beta$. Assim, $\beta \in \overline{\mathbb{Q}}$, o que é um absurdo. Portanto, $\beta^{-1} \in \mathbb{T}$.

Ademais, temos um outro resultado interessante, o chamado Teorema de Gelfond-Schneider, que classifica a natureza aritmética de certas potências de algébricos por algébricos. Foi provado independentemente pelos matemáticos Alexander Gelfond (1906-1968) e Theodor Schneider (19111988), a partir de um dos 23 problemas propostos por David Hilbert (1862-1943), no Segundo Congresso Internacional de Matemática, realizado em Paris.

Teorema 4. (Gelfond-Schneider) Sejam $\alpha$ e $\beta$ dois números algébricos, com $\alpha \neq 0, \alpha \neq 1$ e $\beta$ não sendo um racional. Então $\alpha^{\beta}$ é transcendente.

Demonstração. Ver [6].

Esse teorema traz como caso particular a transcendência dos números $2^{\sqrt{2}}$ e $\log _{10} 2$.

No caso de $2^{\sqrt{2}}$, vale destacar que se tirássemos as hipóteses sobre $\alpha$, o teorema poderia perder sua validade. Pois, por um lado $2^{\sqrt{2}}$ é transcendente, mas, em contrapartida,

$$
\left(2^{\sqrt{2}}\right)^{\sqrt{2}}=4
$$

que é algébrico. 
A justificativa para $\log _{10} 2$ é relativamente simples, pois tomando $\alpha=10$ e $\beta=\log _{10} 2$; e aplicando a definição de logaritmo decimal, temos que:

$$
\alpha^{\beta}=10^{\log _{10} 2}=2 .
$$

Nesse caso, se $\beta=\log _{10} 2$ fosse irracional algébrico, pelo Teorema de Gelfond-Schneider, 2 seria transcendente, o que é absurdo. Logo, $\log _{10} 2$ é transcendente.

Uma outra consequência do referido teorema é a transcendência de $e^{\pi}$, a chamada Constante de Gelfond. De fato,

$$
e^{\pi}=\left(e^{i \pi}\right)^{(-i)}=(-1)^{-i},
$$

ou seja,

$$
e^{\pi}=(-1)^{-i}
$$

que é transcendente.

O curioso é que para potências de números transcendentes por transcendentes ainda não existe nenhum resultado análogo ao de Gelfond e Schneider, mas sabemos que tais potências tanto podem resultar em um número algébrico como em um número transcendente.

Vejamos abaixo, em ordem cronológica de descoberta, alguns números transcendentes:

1873 HERMITE, ver [1] e [2].

Base dos logaritmos neperianos: $e=2,718281828459045 \ldots$

1882 LINDEMANN, ver [1] e [2].

Razão entre a circunferência e o diâmetro de um círculo: $\pi=3,1415926535897 \ldots$

1929 MAHLER, ver [1] e [9].

Sequência de Prouhet-Thue-Morse: $\tau=0,0110101001 \ldots$

1961 MAHLER, ver [1] e [7].

Constante de Champernowne: $c=0,12345678910111213 \ldots$

Convém destacar que existe ainda uma série de números como, $e+\pi, e-\pi, e \pi, \frac{\pi}{e}, e^{e}, \pi^{e}, \pi^{\pi}, 2^{e}$, $2^{\pi}$ entre outros, sobre os quais nada se conhece acerca de sua natureza aritmética.

\section{Números de Liouville}

Nesta seção, apresentaremos importantes resultados da teoria que circunda a classe dos Números de Liouville. As referências aqui utilizadas foram [6], [7], [11] e [9].

Definição 3. Um número algébrico $\alpha$ é dito de grau $n$ se ele for raiz de uma equação polinomial com coeficientes inteiros de grau $n$ e não existir nenhum polinômio com coeficientes inteiros, de grau menor que $n$, que contenha $\alpha$ como uma de suas raízes. 
Exemplo 3. Todo número racional $\frac{p}{q},(p, q \in \mathbb{Z}, q \neq 0)$, é algébrico de grau 1 , pois qualquer número racional $\alpha=\frac{p}{q}$ é raiz da equação $q x-p=0$. Isso implica que, se $\alpha$ é algébrico de grau $\geq 2$, então, $\alpha$ é irracional.

Definição 4. Um número real $\alpha$ é aproximável na ordem $n$ por racionais se existirem uma constante real $c>0$ e uma sequência $\left\{\frac{p_{j}}{q_{j}}\right\}_{j \in \mathbb{N}}$ de racionais distintos, com $q_{j}>0$ e $m d c\left(p_{j}, q_{j}\right)=1$, tais que:

$$
\left|\alpha-\frac{p_{j}}{q_{j}}\right|<\frac{c}{q_{j}^{n}}
$$

É interessante observar que o importante da Definição 4 não é apresentar a existência de uma sucessão de racionais convergindo para $\alpha$, pois tais sucessões sempre existem qualquer que seja o número real $\alpha$. O mais importante que a definição nos traz é que uma sucessão particular de racionais converge para $\alpha$ de certo modo, isto é, de acordo com (3). Ainda na Definição 4, é importante que se tomem os racionais todos diferentes, o que implica que podemos tomá-los diferentes de $\alpha$, mesmo no caso de $\alpha$ ser racional.

O próximo teorema descreve uma importante propriedade de números algébricos. Salientamos que se um certo número não possui uma propriedade que todos os algébricos devem possuir, então ele é transcendente. Isso mostra que o estudo de números algébricos permite também encontrarmos condições para que um número não seja algébrico.

Teorema 5. Seja a um número algébrico real de grau $n>1$, então existe $A>0$, que depende de $\alpha$ tal que

$$
\left|\alpha-\frac{p}{q}\right|>\frac{A}{q^{n}}
$$

para todo racional $\frac{p}{q}$.

Demonstração. Considere $\alpha$ solução de uma equação polinomial da forma:

$$
f(x)=a_{n} x^{n}+a_{n-1} x^{n-1}+\cdots+a_{1} x+a_{0} .
$$

Seja $d>0$ tal que, no intervalo $[\alpha-d, \alpha+d]$ a única raiz de $f(x)=0$ é $\alpha$ (isso deve-se ao fato de que a equação polinomial tem no máximo $n$ raízes reais; portanto, $d$ pode ser qualquer número menor que a menor das distâncias de $\alpha$ às demais raízes reais). Em seguida, observemos que a derivada $f^{\prime}(x)$ de $f(x)$ é um polinômio de grau $n-1$ e, portanto, ela é limitada em intervalos limitados. Para tanto, existe $M>0$ tal que

$$
\left|f^{\prime}(x)\right| \leq M, \text { para } x \in[\alpha-d, \alpha+d] .
$$

Para todo racional $\frac{p}{q}$, com $q \neq 0$, em $[\alpha-d, \alpha+d]$; aplicando o Teorema do Valor Médio, temos:

$$
f(\alpha)-f\left(\frac{p}{q}\right)=f^{\prime}(\psi)\left(\alpha-\frac{p}{q}\right),
$$


onde $\psi \in(\alpha-d, \alpha+d)$.

Como $f(\alpha)=0,(5)$ pode ser escrito como

$$
-f\left(\frac{p}{q}\right)=f^{\prime}(\psi)\left(\alpha-\frac{p}{q}\right)
$$

daí,

$$
\left|f\left(\frac{p}{q}\right)\right| \leq M\left|\alpha-\frac{p}{q}\right|
$$

mas $f(x)=a_{n} x^{n}+a_{n-1} x^{n-1}+\cdots+a_{1} x+a_{0}$, portanto

$$
\left|f\left(\frac{p}{q}\right)\right|=\left|\frac{a_{n} p^{n}+a_{n-1} p^{n-1} q+\cdots+a_{1} p q^{n-1}+a_{0} q^{n}}{q^{n}}\right| \geq \frac{1}{q^{n}},
$$

uma vez que o numerador $a_{n} p^{n}+a_{n-1} p^{n-1} q+\cdots+a_{1} p q^{n-1}+a_{0} q^{n}$ é inteiro e não nulo, e portanto é maior do que 1.

Isso posto, substituindo em (6), temos:

$$
\begin{gathered}
\frac{1}{q^{n}} \leq\left|f\left(\frac{p}{q}\right)\right| \leq M\left|\alpha-\frac{p}{q}\right| \\
\Rightarrow \frac{1}{M q^{n}} \leq \frac{1}{M}\left|f\left(\frac{p}{q}\right)\right| \leq\left|\alpha-\frac{p}{q}\right|
\end{gathered}
$$

implicando

$$
\frac{1}{M q^{n}} \leq\left|\alpha-\frac{p}{q}\right|
$$

ou seja,

$$
\left|\alpha-\frac{p}{q}\right| \geq \frac{1}{M q^{n}}
$$

para $\frac{p}{q} \in[\alpha-d, \alpha+d]$. Se $\frac{p}{q}$ não estiver nesse intervalo, então:

$$
\left|\alpha-\frac{p}{q}\right|>d .
$$

Como $q \geq 1$ temos $q^{n} \geq 1 \Rightarrow \frac{1}{q^{n}} \leq 1 \Rightarrow \frac{d}{q^{n}} \leq d$, daí (7) fica

$$
\left|\alpha-\frac{p}{q}\right|>\frac{d}{q^{n}} \text {. }
$$

Tomando, finalmente, $A$ como o menor dos números $\frac{1}{M}$ e $d$, e notando que

$$
\left(\frac{1}{M} \geq A \text { e d } \geq A\right) \Rightarrow\left(\frac{1}{M q^{n}} \geq \frac{A}{q^{n}} \text { e } \frac{d}{q^{n}} \geq \frac{A}{q^{n}}\right)
$$

chegamos à relação (4) para todo racional $\frac{p}{q}$. 
Sabemos que o conjunto dos números racionais é denso em $\mathbb{R}$. Logo, é possível aproximar qualquer número real por números racionais. Contudo, o Teorema 5 , também conhecido como Teorema de Liouville, afirma que números algébricos (reais) não racionais não podem ser muito "bem aproximados" por racionais, no sentido em que qualquer aproximação tem que respeitar o comportamento descrito pela desigualdade (4).

Por conseguinte, qualquer número que não satisfaça o Teorema 5 não pode ser algébrico, e portanto será transcendente.

Após demonstrar o Teorema 5, Liouville apresentou um conjunto de números que não satisfazem o mesmo. Esses são os números de Liouville e seu conjunto é denodato por $\mathbb{L}$, dados pela definição abaixo.

Definição 5. Um número real $\alpha$ é chamado um número de Liouville se existir uma sequência $\left\{\frac{p_{j}}{q_{j}}\right\}_{j \in \mathbb{N}}$ de racionais distintos, onde $p_{j}, q_{j} \in \mathbb{Z}, \operatorname{com} q_{j}>0$ e $m d c\left(p_{j}, q_{j}\right)=1$, tais que satisfaçam

$$
\left|\alpha-\frac{p_{j}}{q_{j}}\right|<\frac{1}{q_{j}^{j}}
$$

Observe que a potência de $q_{j}$ é $j$, ou seja, é um valor variável e não fixo como na Definição 4 . Além disso, o numerador da fração do lado direito é o valor $c=1$.

A ideia de Liouville ao elaborar a Definição 5 foi apresentar uma classe de números que são bem aproximados por racionais. Em outras palavras, a definição afirma que os números de Liouville são aproximados por racionais tais que o erro dessa aproximação é menor que $\frac{1}{q_{j}^{j}}$. Dessa forma, podemos inferir que os números de Liouville são "muito bem" aproximáveis na ordem $n$ por racionais.

Proposição 4. A sequência $\left\{q_{j}\right\}_{j \geq 1}$ é ilimitada.

Demonstração. Veja que

$$
\left|\alpha-\frac{p_{j}}{q_{j}}\right|<\frac{1}{q_{j}^{j}}<1
$$

Suponha que a sequência $\left\{q_{j}\right\}_{j \geq 1}$ é limitada, isto é, $q_{j} \leq M$.

Multiplicando a equação (9) por $q_{j}$, obtemos:

$$
\left|\alpha q_{j}-p_{j}\right|<q_{j} \leq M
$$

Aplicando a desigualdade triangular diminuída ${ }^{1}$, teremos:

$$
\begin{gathered}
\left|p_{j}\right|-\left|\alpha q_{j}\right| \geq M \\
\left|p_{j}\right| \geq M+|\alpha|\left|q_{j}\right| \geq(|\alpha|+1) M
\end{gathered}
$$

${ }^{1} \mathrm{~A}$ desigualdade triangular diminuída afirma que $|a-b| \geq|a|-|b|$. 
Logo, a sequência $p_{j}$ também é limitada. Absurdo! Pois, existem infinitos $\frac{p_{j}}{q_{j}}$ 's. Dessa forma, $\left\{q_{j}\right\}$ é ilimitada.

Proposição 5. Todo número de Liouville é irracional.

Demonstração. Suponha que $\alpha \in \mathbb{L}$, tal que $\alpha=\frac{p}{q} \in \mathbb{Q}$. Por hipótese, existe uma sequência $\left\{\frac{p_{j}}{q_{j}}\right\}$ de racionais distintos, todos diferentes de $\frac{p}{q}$, tais que

$$
\left|\frac{p}{q}-\frac{p_{j}}{q_{j}}\right|<\frac{1}{q_{j}^{j}}
$$

ou seja,

$$
\left|\frac{p q_{j}-p_{j} q}{q q_{j}}\right|<\frac{1}{q_{j}^{j}}
$$

Além disso, como $p q_{j}$ e $p_{j} q$ são números inteiros distintos, a diferença em módulo é maior ou igual que 1, e assim obtemos:

$$
\left|p q_{j}-p_{j} q\right| \geq 1 \Rightarrow \frac{1}{q q_{j}} \leq\left|\frac{p q_{j}-p_{j} q}{q q_{j}}\right|<\frac{1}{q_{j}^{j}} .
$$

Isso nos diz que $|q| q_{j}>q_{j}^{j}$ e, portanto $|q|>q_{j}^{j-1}$, um absurdo pois $q_{j}^{j-1} \rightarrow \infty$ quando $j \rightarrow \infty$. Logo, todo número de Liouville é irracional.

Teorema 6. Todo número de Liouville é transcendente.

Demonstração. De acordo com a Proposição 5 temos que um número de Liouville não pode ser racional. Suponha que $\alpha$ seja um número de Liouville algébrico não racional, ou seja, de grau $n \geq 2$. Então, pelo Teorema 5 segue-se que a relação (4) será válida para todo número racional. Em particular, para os $\frac{p_{j}}{q_{j}}$ da Definição 5 . Desse modo, teríamos

$$
\frac{A}{q^{n}}<\left|\alpha-\frac{p_{j}}{q_{j}}\right|<\frac{1}{q_{j}^{j}}
$$

ou melhor,

$$
\frac{A}{q^{n}}<\frac{1}{q_{j}^{j}} \Rightarrow \frac{q_{j}^{j}}{q^{n}}<\frac{1}{A} \Rightarrow q^{j-n}<\frac{1}{A}, \forall j \in \mathbb{N} .
$$

O que é uma contradição, pois $q^{j-n} \rightarrow \infty$. Logo, $\alpha$ não pode ser algébrico, o que resulta em $\alpha \in \mathbb{T}$. 
Desta forma, subsidiado pelos resultados apresentados, Liouville explicitou o primeiro exemplo de número transcendente, a chamada Constante de Liouville.

Exemplo 4. Considere o número definido por

$$
\alpha=\sum_{k=1}^{\infty} \frac{1}{10^{k !}}=0.110001000000000000000001 \ldots
$$

então, $\alpha$ é transcendente.

Conforme evidenciado anteriormente, basta mostrar que $\alpha$ é um número de Liouville. Para tanto, defina $m_{j}=\sum_{k=1}^{j} 10^{(j !-k !)}$ e $n_{j}=10^{j !}>0$. Observe que $m_{j}, n_{j} \in \mathbb{Z}$, para todo $j \in \mathbb{N}$ e $\frac{m_{j}}{n_{j}}=$ $\frac{\sum_{k=1}^{j} 10^{(j !-k !)}}{10^{j !}}=\sum_{k=1}^{j} 10^{-k !}=\sum_{k=1}^{j} \frac{1}{10^{k !}}$.

Assim,

$$
\begin{aligned}
\left|\alpha-\frac{m_{j}}{n_{j}}\right|=\left|\sum_{k=1}^{\infty} \frac{1}{10^{k !}}-\sum_{k=1}^{j} \frac{1}{10^{k !}}\right| \\
=\sum_{k=1+j}^{\infty} \frac{1}{10^{k !}}=\frac{1}{10^{(1+j) !}}+\frac{1}{10^{(2+j) !}}+\frac{1}{10^{(3+j) !}}+\ldots \\
=\frac{1}{10^{(1+j) !}}\left(1+\frac{1}{10^{(2+j) !-(1+j) !}}+\frac{1}{10^{(3+j) !-(1+j) !}}+\ldots\right) .
\end{aligned}
$$

Agora veja que

$$
\begin{gathered}
=\frac{1}{10^{(1+j) !}}\left(1+\frac{1}{10^{(2+j) !-(1+j) !}}+\frac{1}{10^{(3+j) !-(1+j) !}}+\ldots\right) \\
<\frac{1}{10^{(1+j) !}}\left(1+\frac{1}{10}+\frac{1}{10^{2}}+\frac{1}{10^{3}}+\ldots\right)
\end{gathered}
$$

Observe que

$$
\left(1+\frac{1}{10}+\frac{1}{10^{2}}+\frac{1}{10^{3}}+\ldots\right)=\left(1+\frac{1}{9}\right)=\frac{10}{9},
$$

pois corresponde à soma infinita dos termos de uma progressão geométrica.

Daí, temos que

$$
\left|\alpha-\frac{m_{j}}{n_{j}}\right|<\frac{1}{10^{(1+j) !}} \cdot \frac{10}{9} .
$$

Como $(1+j) !=(1+j) j !=j !+j j !$, temos $\frac{1}{10^{(1+j) !}}=\frac{1}{10^{j !}\left(10^{j !}\right)^{j}}$.

Logo,

$$
\left|\alpha-\frac{m_{j}}{n_{j}}\right|<\frac{10}{9 \cdot 10^{(1+j) !}}<\frac{1}{\left(10^{j !}\right)^{j}}
$$

e como $n_{j}=10^{j !}$, obtemos

$$
\left|\alpha-\frac{m_{j}}{n_{j}}\right|<\frac{1}{n_{j}^{j}}
$$

O que implica que $\alpha$ é número de Liouville. Logo, pelo Teorema 6, $\alpha$ é um número transcendente. 
Com o avanço dos métodos computacionais, constatou-se que, por uma margem de erro extremamente pequena, a constante de Liouville não satisfaz a equação:

$$
10 x^{6}-76 x^{3}-190 x+21=0
$$

pois, substituindo a indeterminada $x$ pela constante de Liouville, $0.1100010 \ldots$ a equação dará -0.0000000059 muito próximo de ZERO.

Podemos generalizar o Exemplo 4 acima e construir infinitos outros números de Liouville dandonos uma quantidade não enumerável, exibindo assim infinitos números transcendentes distintos, como pode ser visto no exemplo abaixo.

Exemplo 5. Os números da forma

$$
\alpha=\sum_{k=1}^{\infty} \frac{a_{k}}{10^{k !}}=0 . a_{1} a_{2} 000 a_{3} 00000000000000000 a_{4} \ldots,
$$

onde $a_{k} \in\{1,2,3,4,5,6,7,8,9\}$ para todo $k \in \mathbb{N}$ são números transcendentes.

Considere os números inteiros $m_{j}=\sum_{k=1}^{j} a_{k} 10^{j !-k !}$ e $n_{j}=10^{j !}>0$ com $k \in \mathbb{N}$. Observe $\frac{m_{j}}{n_{j}}=$ $\frac{\sum_{k=1}^{j} a_{k} 10^{(j !-k !)}}{10^{j !}}=\sum_{k=1}^{j} \frac{a_{k} 10^{j !}}{10^{k !} 10^{j !}}=\sum_{k=1}^{j} \frac{a_{k}}{10^{k !}}$.

Assim,

$$
\begin{gathered}
\left|\alpha-\frac{m_{j}}{n_{j}}\right|=\left|\sum_{k=1}^{\infty} \frac{a_{k}}{10^{k !}}-\sum_{k=1}^{j} \frac{a_{k}}{10^{k !}}\right| \\
=\sum_{k=1+j}^{\infty} \frac{a_{k}}{10^{k !}}=\frac{a_{1+j}}{10^{(1+j) !}}+\frac{a_{2+j}}{10^{(2+j) !}}+\frac{a_{3+j}}{10^{(3+j) !}}+\ldots \\
=\frac{1}{10^{(1+j) !}}\left(a_{1+j}+\frac{a_{2+j}}{10^{(2+j) !-(1+j) !}}+\frac{a_{3+j}}{10^{(3+j) !-(1+j) !}}+\ldots\right) \\
\leq \frac{9}{10^{(1+j) !}}\left(1+\frac{1}{10^{(2+j) !-(1+j) !}}+\frac{1}{10^{(3+j) !-(1+j) !}}+\ldots\right) .
\end{gathered}
$$

Analogamente ao que fizemos no Exemplo 4, temos

$$
\frac{9}{10^{(1+j) !}}\left(1+\frac{1}{10^{(2+j) !-(1+j) !}}+\frac{1}{10^{(3+j) !-(1+j) !}}+\ldots\right) \leq \frac{9}{10^{(1+j) !}} \cdot \frac{10}{9}=\frac{10}{10^{j !}\left(10^{j !}\right)^{j}} .
$$

Daí,

$$
\left|\alpha-\frac{m_{j}}{n_{j}}\right| \leq \frac{10}{10^{j !}\left(10^{j !}\right)^{j}}<\frac{1}{\left(10^{j !}\right)^{j}}
$$

no que implica que

$$
\left|\alpha-\frac{m_{j}}{n_{j}}\right|<\frac{1}{n_{j}^{j}}
$$

uma vez que $n_{j}^{j}=10^{j !}$.

Como, pelo Teorema 6, todo número de Liouville é transcendente, segue que $\alpha$ é transcendente. 
Vale observar que o Exemplo 5 pode ser generalizado para números em qualquer base, ou seja, que qualquer número da forma

$$
\sum_{j>1} \frac{a_{j}}{k^{j !}}
$$

com $k \in \mathbb{N}-\{1\}$ e $a_{j} \in\{0,1,2, \ldots, k-1\}$, também é número de Liouville.

A priori, agora sabemos mais do que apenas a existência de números transcendentes, podemos, de fato, exibi-los.

Observamos, por fim, que a recíproca do Teorema 6 não é válida. Na verdade, é possível provar que quase nenhum número transcendente é número de Liouville (A demonstração desse fato pode ser encontrada em [11]). Citando um exemplo, provou-se, com a ajuda de resultados merecedores (e ganhadores) da medalha Fields (veja [8]), que a constante de Champernowne dada por $c=$ $0.1234567891011121314 \ldots$ é um número transcendente. Apesar disso, $c$ não é um número de Liouville. Os números $e$ e $\pi$, apesar de serem transcendentes, também não são números de Liouville.

Um resultado surpreendente, demonstrado por Paul Erdös (1913-1996) em 1962, é o fato de que todo número real pode ser escrito como soma de dois números de Liouville. Para mostrar essa afirmação precisaremos de alguns resultados preliminares que se encontram abaixo.

Lema 1. $\alpha \in \mathbb{L}$ se, e somente, se para todo $n \in \mathbb{N}$ existe $\frac{p}{q} \in \mathbb{Q}$ tal que

$$
0<\left|\alpha-\frac{p}{q}\right|<\frac{1}{q^{n}}
$$

Demonstração. Se $\alpha$ é um número de Liouville, dado $n \in \mathbb{N}$, podemos tomar $p=p_{n}$ e $q=q_{n}$. Reciprocamente, dado $n \in \mathbb{N}$, vamos escolher $\frac{p_{n}}{q_{n}} \in \mathbb{Q}$ de modo que $q_{n}>1 \mathrm{e}$

$$
0<\left|\alpha-\frac{p_{n}}{q_{n}}\right|<\frac{1}{q_{n}^{n}}
$$

Seja

$$
A=\bigcup_{n \geq 1}\left\{\frac{p_{n}}{q_{n}}\right\}
$$

Se $A$ for finito, então existe $\frac{p}{q} \in A$ tal que $|\alpha-p / q|<q^{-n}$ para $n \in \mathbb{N}^{\prime}$, com $\mathbb{N}^{\prime} \subset \mathbb{N}$ infinito. Assim, $\alpha=\frac{p}{q}$, contradizendo $|\alpha-p / q|>0$. Portanto, $A$ é infinito. Concluímos que $\alpha$ é um número de Liouville.

Lema 2. Dado $\alpha \in \mathbb{R}$, se existirem $c>0$ e uma sequência de racionais $\left\{\frac{p_{j}}{q_{j}}\right\}_{j \geq 1}$ com $q_{j} \geq 1$ tais que

$$
0<\left|\alpha-\frac{p_{j}}{q_{j}}\right|<\frac{c}{q_{j}^{j}},
$$

então $\alpha \in \mathbb{L}$. 
Demonstração. Se $0<c \leq 1$ então

$$
\left|\alpha-\frac{p_{j}}{q_{j}}\right|<\frac{c}{q_{j}^{j}} \leq \frac{1}{q_{j}^{j}}
$$

e segue o resultado.

Se $c>1$, observe que a sequência $q_{j}$ é ilimitada. Dessa forma, existe $j_{0}$ tal que para todo $j>j_{0}$ tem-se que $c<q_{j}^{j_{0}}$ donde

$$
\left|\alpha-\frac{p_{j}}{q_{j}}\right|<\frac{c}{q_{j}^{j}}<\frac{q_{j}^{j_{0}}}{q_{j}^{j}}=\frac{1}{q_{j}^{j-j_{0}}}, \forall j \geq j_{0} .
$$

Para todo $j \geq j_{0}$, considere a sequência dos números naturais $n=j-j_{0}$. Desse modo, temos que para todo $n \in \mathbb{N}$ existe $\frac{p}{q}=\frac{p_{j}}{q_{j}}$ tal que

$$
\left|\alpha-\frac{p_{j}}{q_{j}}\right|<\frac{1}{q^{n}}
$$

e pelo Lema 1 temos que $\alpha$ é um número de Liouville.

Lema 3. Se existirem uma sequência ilimitada $\left\{w_{k}\right\}_{k \geq 1}$ de números reais positivos e uma sequência de racionais $\left\{\frac{p_{k}}{q_{k}}\right\}_{k \geq 1}$ tais que

$$
0<\left|\alpha-\frac{p_{k}}{q_{k}}\right|<\frac{1}{q_{k}^{w_{k}}}, \forall k \in \mathbb{N},
$$

então $\alpha \in \mathbb{L}$.

Demonstração. Seja $\left\{s_{k}\right\}_{k \geq 1}$ contida em $\left\{w_{k}\right\}_{k \geq 1}$ uma subsequência ilimitada em que $s_{k}>1$ para todo $k \in \mathbb{N}$ e $\left\{\frac{a_{k}}{b_{k}}\right\}_{k \geq 1}$ contida em $\left\{\frac{p_{k}}{q_{k}}\right\}_{k \geq 1}$ tais que

$$
0<\left|\alpha-\frac{a_{k}}{b_{k}}\right|<\frac{1}{b_{k}^{s_{k}}}, \forall k \in \mathbb{N} .
$$

Agora tome uma sequência de números reais $\left\{r_{k}\right\}_{k \geq 1} \operatorname{com} r_{k}>0$ para todo $k \in \mathbb{N}$ tal que $s_{k}-r_{k}=k$, então

$$
0<\left|\alpha-\frac{a_{k}}{b_{k}}\right|<\frac{1}{b_{k}^{s_{k}}}<\frac{1}{b_{k}^{s_{k}-r_{k}}}=\frac{1}{b_{k}^{k}}, \forall k \in \mathbb{N} .
$$

Logo, pelo Lema 1, segue o resultado.

Teorema 7. Se $\alpha \in \mathbb{L}$ e $\frac{p}{q} \in \mathbb{Q}^{*} \operatorname{com} q \geq 1$, então

(i) $\alpha \frac{p}{q} \in \mathbb{L}$; 
(ii) $\left(\alpha+\frac{p}{q}\right) \in \mathbb{L}$.

Demonstração. Se $\alpha \in \mathbb{L}$, então existe uma sequência de racionais $\left\{\frac{p_{j}}{q_{j}}\right\}_{j \geq 1}$ tal que

$$
\left|\alpha-\frac{p_{j}}{q_{j}}\right|<\frac{1}{q_{j}^{j}}
$$

(i) Seja $\alpha_{j}=\log _{q} q_{j}$ donde $q^{\alpha_{j}}=q_{j}$. Observe que $\lim _{j \rightarrow \infty} \alpha_{j}=+\infty$ e ainda

$$
\left|\alpha \frac{p}{q}-\frac{p}{q} \frac{p_{j}}{q_{j}}\right|=\left|\alpha \frac{p}{q}-\frac{p p_{j}}{q q^{\alpha_{j}}}\right|=\left|\alpha \frac{p}{q}-\frac{p p_{j}}{q^{\alpha_{j}+1}}\right| .
$$

Por outro lado, observe que existe $k \in \mathbb{R}$ tal que $|p|<q^{k}$, então

$$
\begin{gathered}
\left|\alpha \frac{p}{q}-\frac{p p_{j}}{q q_{j}}\right|=\frac{|p|}{q}\left|\alpha-\frac{p_{j}}{q_{j}}\right|<\frac{|p|}{q} \frac{1}{q_{j}^{j}}= \\
=\frac{|p|}{q\left(q^{\left.\alpha_{j}\right)^{j}}\right.}=\frac{|p|}{q^{j \alpha_{j}+1}}=\frac{|p|}{\left(q^{\alpha_{j}+1}\right)^{\frac{j \alpha_{j}+1}{\alpha_{j}+1}}} \\
<\frac{q^{k}}{\left(q^{\alpha_{j}+1}\right)^{\frac{j \alpha_{j}+1}{\alpha_{j}+1}}}=\frac{1}{\left(q^{\alpha_{j}+1}\right)^{\frac{j \alpha_{j}+1}{\alpha_{j}+1}-k}} .
\end{gathered}
$$

Agora, sabemos pelo Cálculo Diferencial e Integral que se uma função $f(x)$ é ilimitada, então, pelo Teorema de L'hospital, $\lim _{x \rightarrow \infty} \frac{f(x)}{1+f(x)}=1$, e, assim, $\lim _{x \rightarrow \infty} x \frac{f(x)}{1+f(x)}=+\infty$. Logo,

$$
\lim _{j \rightarrow \infty}\left(\frac{j \alpha_{j}+1}{\alpha_{j}+1}-k\right)=+\infty
$$

e segue pelo Lema 3 que $\alpha \frac{p}{q} \in \mathbb{L}$.

(ii) Como no item anterior

$$
\begin{aligned}
& \left|\left(\alpha+\frac{p}{q}\right)-\left(\frac{p_{j}}{q_{j}}+\frac{p}{q}\right)\right|=\left|\left(\alpha+\frac{p}{q}\right)-\left(\frac{p_{j} q+q_{j} p}{q q_{j}}\right)\right| \\
= & \left|\left(\alpha+\frac{p}{q}\right)-\left(\frac{p_{j} q+q_{j} p}{q q^{\alpha_{j}}}\right)\right|=\left|\left(\alpha+\frac{p}{q}\right)-\left(\frac{p_{j} q+q_{j} p}{q^{\alpha_{j}+1}}\right)\right| .
\end{aligned}
$$

Por outro lado,

$$
\begin{aligned}
& \left|\left(\alpha+\frac{p}{q}\right)-\left(\frac{p_{j}}{q_{j}}+\frac{p}{q}\right)\right|=\left|\alpha-\frac{p_{j}}{q_{j}}\right| \\
& <\frac{1}{q_{j}^{j}}=\frac{1}{\left(q^{\alpha_{j}}\right)^{j}}=\frac{1}{q^{j \alpha_{j}}}=\frac{1}{\left(q^{\alpha_{j}+1}\right)^{\frac{j \alpha_{j}}{\alpha_{j}+1}}} .
\end{aligned}
$$

Mas, já sabemos que $\lim _{j \rightarrow+\infty} \frac{j \alpha_{j}}{\alpha_{j}+1}=+\infty$, donde, pelo Lema 3 segue que $\left(\alpha+\frac{p}{q}\right) \in \mathbb{L}$. 
Para tanto, segue o Teorema de Erdös que nos afirma que qualquer número real pode ser escrito como soma de dois números de Liouville.

Teorema 8. Dado $\beta \in \mathbb{R}$, então existem $l_{1}, l_{2} \in \mathbb{L}$ tais que $\beta=l_{1}+l_{2}$.

Demonstração. De fato, se $\beta \in \mathbb{L}$, então pelo Teorema 7 basta tomar $l_{1}=l_{2}=\frac{\beta}{2}$.

Se $\beta \in \mathbb{Q}$ tomemos qualquer $\alpha \in \mathbb{L}$ pelo Teorema $7\left(\frac{\beta+\alpha}{2}\right)$ e $\left(\frac{\beta-\alpha}{2}\right) \in \mathbb{L} \operatorname{com} \beta=\frac{\beta-\alpha}{2}+\frac{\beta+\alpha}{2}$.

Agora, se $\beta \notin \mathbb{Q}$, então $(\beta-[\beta]) \notin \mathbb{Q}$, onde $[\beta]$ é a parte inteira de $\beta$, e assim podemos estudar apenas o caso em que $\beta \in(0,1) \cap \mathbb{Q}^{\mathbb{Q}}$. Seja

$$
\beta=0 . a_{1} a_{2} a_{3} \ldots a_{n}
$$

onde $a_{n} \in\{0,1,2,3, \ldots, 9\}$ e difina

$$
l_{1}=\sum_{n=1}^{\infty} \frac{\lambda_{n}}{10^{n}} \text { e } l_{2}=\sum_{n=1}^{\infty} \frac{\delta_{n}}{10^{n}}
$$

em que para $n ! \leq k \leq(n+1)$ ! temos

$$
\begin{gathered}
\lambda_{k}=a_{k} \text { e } \delta_{k}=0 \text { se } n=1,3,5, \ldots \\
\delta_{k}=a_{k} \text { e } \lambda=0 \text { se } n=2,4,6, \ldots
\end{gathered}
$$

Note que $\beta=l_{1}+l_{2}$. Falta mostrar que $l_{1}$ e $l_{2} \in \mathbb{L}$. Para $l_{1}$, tomemos $p_{n}=\sum_{k-1}^{(2 n) !-1} \lambda_{k} 10^{(2 n) !-(1+k)}$ e $q_{n}=10^{(2 n) !-1}$, daí

$$
\frac{p_{n}}{q_{n}}=\frac{\sum_{k-1}^{(2 n) !-1} \lambda_{k} 10^{(2 n) !-(1+k)}}{10^{(2 n) !-1}}=\sum_{k-1}^{(2 n) !-1} \frac{\lambda_{k} 10^{(2 n) !-1}}{10^{k} 10^{(2 n) !-1}}=\sum_{k-1}^{(2 n) !-1} \frac{\lambda_{k}}{10^{k}} .
$$

Segue-se que

$$
\left|l_{1}-\frac{p}{q}\right|=\sum_{k=(2 n) !}^{\infty} \frac{\lambda_{k}}{10^{k}}
$$

Como $\lambda_{k}=0$ para $(2 n) ! \leq k<(2 n+1)$ ! podemos escrever

$$
\begin{gathered}
\left|l_{1}-\frac{p}{q}\right|=\sum_{k=(2 n+1) !}^{\infty} \frac{\lambda_{k}}{10^{k}} \leq \sum_{k=(2 n+1) !}^{\infty} \frac{9}{10^{k}}= \\
=\frac{9}{10^{(2 n) !+1}}\left(1+\frac{1}{10}+\frac{1}{10^{2}}+\ldots\right)=\frac{9}{10^{(2 n) !+1}} \cdot \frac{10}{9}=\frac{1}{10^{((2 n) !+1)-1}} \\
<\frac{1}{10^{n((2 n) !-1)}}=\frac{1}{\left(10^{(2 n) !-1)^{n}}\right.}=\frac{1}{q_{n}^{n}} .
\end{gathered}
$$

Logo, $l_{1} \in \mathbb{L}$. De modo análogo pode-se provar que $l_{2} \in \mathbb{L}$.

Esse resultado é bem curioso, visto que é possível mostrar que o conjunto dos números de Liouville tem medida nula ${ }^{2}$ em $\mathbb{R}$, isto é, quase nenhum número real é de Liouville. Isso posto, podemos pensar então que, mesmo sendo um conjunto "invisível", os números de Liouville estão estrategicamente posicionados na reta real [8].

\footnotetext{
${ }^{2}$ Ao leitor interessado sobre esse assunto recomendamos a leitura de [11].
} 


\section{Considerações finais}

O desenvolvimento do presente trabalho possibilitou apresentar um estudo sobre tópicos que compreendem a Teoria dos Números Transcendentes; uma área da Teoria dos Números em que desde o século XVII especulam-se questões relacionadas à natureza transcendental dos números a partir do estudo de equações polinomiais, em específico, suas raízes, a saber, números algébricos. Esta teoria foi originada por Joseph Liouvile, ao criar uma classe numérica, os Números de Liouville, pela qual não só provou a existência dos números transcendentes, como também explicitou seus primeiros exemplos.

Ademais, a referida teoria mostra-se viva e convidativa, pois apresenta problemas ainda em aberto e viabiliza investigações relacionadas às suas possíveis aplicabilidades. Nessa perspectiva, o presente trabalho buscou contribuir para seu desenvolvimento teórico ao apresentar a definição e a caracterização do conjunto dos números algébricos e dos números transcendentes. Além disso, elencam-se importantes resultados sobre a classe dos números de Liouville, permitindo, assim, considerarmos que os objetivos propostos foram realmente alcançados.

Nesse sentido, este trabalho apresenta-se como uma pequena pesquisa em Teoria Transcendente, cuja importância reside em subsidiar estudos futuros de modo que coadjuve o seu progresso teórico no meio científico, haja vista tratar-se de área recente e que tem despertado o interesse de muitos pesquisadores.

\section{Referências}

[1] Burger, Edward. Tubbs, Robert. Making transcendence transparent. New York: Springer, 2004.

[2] Figueiredo, D. G. Números irracionais e transcendentes. $3^{\underline{a}}$ ed. Coleção de Iniciação Científica. Rio de Janeiro: SBM, 2011.

[3] Latefá, C. A.; Silva, E.; Lelis, J. Teoria dos Números Transcendentes: do teorema de Liouville à conjectura de Schanuel. 1.ed. VIII Bienal da Sociedade Brasileira de Matemática. Rio de Janeiro/RJ, 2016.

[4] Liouville, J., Remarques relatives à des classes trés-étendues de quantités dont la valeur n'est nialgébrique, ni même réductible à des irrationnelles algébriques, C. R. Acad. Sci. Paris, 18 (1844), 883-885.

[5] Liouville, J., Sur des classes très-étendues de quantités dont la valeur n'est ni algébrique, ni même réductible à des irrationnelles algébriques, Journal de mathématiques pures et appliquées, 16 (1851), no $1,133-142$.

[6] Marques, D. Alguns resultados que geram números transcendentes. Disertação (Mestrado em Matemática). UFC, Fortaleza/CE, 2007.

[7] Marques, D. O problema de Land e uma generalização dos teoremas de Stäckel. Tese (Doutorado em Matemática). UNB, Brasília/DF, 2009.

[8] Marques, D. Teoria dos Números Transcendentes. Coleção Matemática Universitária. Rio de Janeiro: SBM, 2013.

[9] Mascarenhas, S. P. A Irracionalidade e Transcendência dos Números. Dissertação (Mestrado em Matemática). UFC, Fortaleza/CE, 2017. 
[10] Oliveira, D.; Hoyos, M. G. C. Números Transcendentes: Números de Liouville e a Constante de Chapernowne. Dissertação (Mestrado Profissional em Matemática). UFSJ, São João del Rei/ MG, 2015.

[11] Silva, E. C. S. Alguns resultados relacionados a números de Liouville. Dissertação (Mestrado em Matemática). UNB, Brasília/DF, 2015.

[12] Silva, W. D.; CARVAlHO, T. O. A Transcendência da Constante de Thue-Morse. Anais da $31{ }^{a}$ Semana da Matemática UEL, Londrina/PR, 2016.

Lindauriane Vieira

Instituto Federal de Educação, Ciência e Tecnologia do Ceará Canindé/CE $<$ lindaurianevieiramath@gmail.com>

Luiz Feitoza

Universidade Federal do Ceará Fortaleza/CE <au-gustavo@hotmail.com>

Diego Lima

Instituto Federal de Educação, Ciência e Tecnologia do Ceará Maracanaú/CE <poncianodiego@gmail.com>

Genilson da Silva

Instituto Federal de Educação, Ciência e Tecnologia do Ceará Canindé/CE $<$ genilsonmath@gmail.com>

Recebido: 04/05/2018 